PROCEEDINGS OF THE

AMERICAN MATHEMATICAL SOCIETY

Volume 134, Number 9, September 2006, Pages 2633-2637

S 0002-9939(06)08627-8

Article electronically published on April 7, 2006

\title{
A REMARK ON THE EXISTENCE OF SUITABLE VECTOR FIELDS RELATED TO THE DYNAMICS OF SCALAR SEMI-LINEAR PARABOLIC EQUATIONS
}

\author{
FENGBO HANG AND HUIQIANG JIANG
}

(Communicated by David S. Tartakoff)

\begin{abstract}
In 1992, P. Poláčik showed that one could linearly imbed any vector field into a scalar semi-linear parabolic equation on $\Omega$ with Neumann boundary condition provided that there exists a smooth vector field $\Phi=$ $\left(\phi_{1}, \cdots, \phi_{n}\right)$ on $\bar{\Omega}$ such that

$$
\left\{\begin{array}{l}
\operatorname{rank}\left(\Phi(x), \partial_{1} \Phi(x), \cdots, \partial_{n} \Phi(x)\right)=n \text { for all } x \in \bar{\Omega}, \\
\frac{\partial \Phi}{\partial \nu}=0 \text { on } \partial \Omega
\end{array}\right.
$$
\end{abstract}

In this short paper, we give a classification of all the domains on which one may find such a type of vector field.

Let $\Omega$ be a bounded smooth domain in $\mathbb{R}^{n}$, let $\nu$ be the outer normal direction of $\partial \Omega$ and let $f \in C^{\infty}\left(\bar{\Omega} \times \mathbb{R} \times \mathbb{R}^{n}, \mathbb{R}\right)$. The infinite-dimensional dynamical system defined by

$$
\left\{\begin{array}{l}
u_{t}=\Delta u+f(x, u, \nabla u), \quad x \in \Omega, t>0, \\
\frac{\partial u}{\partial \nu}=0 \text { on } \partial \Omega,
\end{array}\right.
$$

on suitable Sobolev spaces has attracted much interest (see $[\mathrm{A}, \mathrm{H}]$ and more recent references at the end of this note). When $n=1$, (1) has rather simple dynamics, and each bounded solution will converge to an equilibrium. The situation is quite different when $n \geq 2$; the solutions of (1) can exhibit very complicated behavior. One relatively easy way to demonstrate the complexity of its dynamical behavior is the realization of ODEs in (11). We refer the reader to the recent survey paper [P4] by P. Poláčik for a quick overview of the progress made up to 2002; more results can

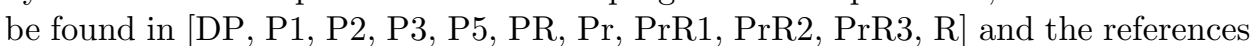
therein.

In particular, the following nice result was proved in $\underline{\mathrm{P} 2}$ : if there exists a smooth vector field $\Phi$ on $\bar{\Omega}, \Phi=\left(\phi_{1}, \cdots, \phi_{n}\right)$ such that

$$
\left\{\begin{array}{l}
\operatorname{rank}\left(\Phi(x), \partial_{1} \Phi(x), \cdots, \partial_{n} \Phi(x)\right)=n \text { for all } x \in \bar{\Omega}, \\
\frac{\partial \Phi}{\partial \nu}=0 \text { on } \partial \Omega
\end{array}\right.
$$

then for any smooth vector field $X$ on $\mathbb{R}^{n}$, there exists a smooth function $f$, such that the linear space span $\left\{\phi_{1}, \cdots, \phi_{n}\right\}$ is invariant under (11) and for any integral curve of $X, c=c(t), u=\sum_{i=1}^{n} c_{i}(t) \phi_{i}(x)$ is a solution to (11). Moreover, it was

Received by the editors March 25, 2005.

2000 Mathematics Subject Classification. Primary 35K20; Secondary 35B40, 54F65.

The research of the first author was supported in part by NSF Grant DMS-0209504.

(C)2006 American Mathematical Society 
shown that this kind of vector field always exists on a starshaped domain. The main result of this short note is a classification of all the domains on which one may find this type of vector field. More precisely, we have

Theorem 1. Let $\Omega \subset \mathbb{R}^{n}(n \geq 2)$ be an open bounded smooth domain. Then the necessary and sufficient condition for the existence of a smooth map $F: \bar{\Omega} \rightarrow \mathbb{R}^{n}$ with

$$
\left\{\begin{array}{l}
\operatorname{rank}\left(F(x), \partial_{1} F(x), \cdots, \partial_{n} F(x)\right)=n \text { for any } x \in \bar{\Omega} \\
\frac{\partial F}{\partial \nu}=0 \text { on } \partial \Omega
\end{array}\right.
$$

is that $\bar{\Omega}$ is diffeomorphic to $\bar{B}_{1}$ or $\bar{B}_{2} \backslash B_{1}$. Here $B_{1}$ and $B_{2}$ are two open balls centered at zero with radius 1 and 2 , respectively.

Remark 1. In fact, if $\bar{\Omega}$ is diffeomorphic to $\bar{B}_{1}$, then any solution to (2), $F$, must have exactly one zero in $\Omega$. If $\bar{\Omega}$ is diffeomorphic to $\bar{B}_{2} \backslash B_{1}$, then any solution to (2), $F$, does not vanish at all. These conclusions will follow from the arguments below.

Remark 2. Our theorem improves the linear imbedding result of P. Poláčik [P2, and at the same time, it exhibits the limitation of the linear realization method. On the other hand, realization of ODEs in a nonlinear fashion can be made in a much more general setting. For example, it is shown in $[\mathrm{P} 3, \mathrm{PrR} 2, \mathrm{PrR} 3$, that any ODE, in any dimension, has an arbitrarily small perturbation that is nonlinearly realizable in a single semi-linear parabolic equation defined on any given open subset of $\mathbb{R}^{n}$, $n \geq 2$.

To prove our main result, we first show that Neumann boundary condition in (2) can be relaxed.

Lemma 1. Let $\Omega \subset \mathbb{R}^{n}(n \geq 2)$ be an open bounded smooth domain. If there exists a smooth map $G: \bar{\Omega} \rightarrow \mathbb{R}^{n}$ such that

$$
\operatorname{rank}\left(G(x), \partial_{1} G(x), \cdots, \partial_{n} G(x)\right)=n \text { for any } x \in \bar{\Omega}
$$

and

$$
\operatorname{dim} \operatorname{span}\left\{G(x), \operatorname{im}\left(\left.G\right|_{\partial \Omega}\right)_{*, x}\right\}=n \text { for any } x \in \partial \Omega
$$

(here $\left(\left.G\right|_{\partial \Omega}\right)_{*, x}$ denotes the tangent map of $\left.G\right|_{\partial \Omega}$ at $\left.x\right)$, then we may find a smooth map $F: \bar{\Omega} \rightarrow \mathbb{R}^{n}$ satisfying (2).

Proof. Let $\varepsilon>0$ be small enough such that the map

$$
\phi: \partial \Omega \times[0,3 \varepsilon] \rightarrow\{y \in \bar{\Omega}: \operatorname{dist}(y, \partial \Omega) \leq 3 \varepsilon\}
$$

defined by

$$
\phi(x, t)=x-t \nu(x)
$$

is a diffeomorphism. Let $P=G \circ \phi$. For any $t \in[0,3 \varepsilon]$, let $P_{t}(x)=P(x, t)$ for $x \in \partial \Omega$. Then we may assume $\varepsilon$ is small enough such that for any $t \in[0,3 \varepsilon]$,

$$
\operatorname{dim} \operatorname{span}\left\{P_{t}(x), \operatorname{im}\left(P_{t}\right)_{*, x}\right\}=n \text { for all } x \in \partial \Omega .
$$

Let $\eta: \mathbb{R} \rightarrow \mathbb{R}$ be a smooth function such that

$$
\eta(t)=\left\{\begin{array}{l}
\varepsilon, \text { when } t \leq \varepsilon / 2, \\
t, \text { when } t \geq 3 \varepsilon / 2,
\end{array}\right.
$$


and $\eta^{\prime}(t) \geq 0$ for all $t$. Define

$$
Q_{t}(x)=Q(x, t)=P(x, \eta(t)) \text { for } x \in \partial \Omega, 0 \leq t \leq 3 \varepsilon .
$$

Then it is clear that for any $t \in[0,3 \varepsilon]$,

$$
\operatorname{dim} \operatorname{span}\left\{Q_{t}(x), \operatorname{im}\left(Q_{t}\right)_{*, x}\right\}=n \text { for any } x \in \partial \Omega \text {. }
$$

Let

$$
F(y)= \begin{cases}G(y) & \text { if } y \in \bar{\Omega}, \operatorname{dist}(y, \partial \Omega) \geq 2 \varepsilon \\ Q\left(\phi^{-1}(y)\right) & \text { if } y \in \bar{\Omega}, \operatorname{dist}(y, \partial \Omega) \leq 3 \varepsilon .\end{cases}
$$

Then it is easy to check that $F$ satisfies all the requirements.

Since the existence of a smooth vector field $G$ in Lemma 1 is a property of the $\bar{\Omega}$ which is preserved under diffeomorphisms, we conclude

Corollary 1. Assume $\bar{\Omega}_{1}$ is diffeomorphic to $\bar{\Omega}_{2}$, and for $\Omega_{1}$ we may find a solution to (2); then we may find a solution to (2) for $\Omega_{2}$, too.

To derive the necessary condition for the existence of a vector field satisfying (2), we will need

Lemma 2. Let $\Omega \subset \mathbb{R}^{n}(n \geq 2)$ be an open bounded smooth domain, if there exists a smooth map $H: \bar{\Omega} \rightarrow S^{n-1}$ such that

$$
\operatorname{rank}\left(\partial_{1} H(x), \cdots, \partial_{n} H(x)\right)=n-1 \text { for any } x \in \bar{\Omega}
$$

and

$$
\operatorname{dim} \operatorname{im}\left(\left.H\right|_{\partial \Omega}\right)_{*, x}=n-1 \text { for any } x \in \partial \Omega .
$$

Then $\bar{\Omega}$ is diffeomorphic to $\bar{B}_{2} \backslash B_{1}$.

Proof. First we claim that each path connected component of $\partial \Omega$ is diffeomorphic to $S^{n-1}$. This is clear when $n=2$. If $n \geq 3$, since $\left.H\right|_{\partial \Omega}$ has full rank everywhere and $\partial \Omega$ is compact,

$$
\left.H\right|_{\partial \Omega}: \partial \Omega \rightarrow S^{n-1}
$$

is a covering map (see [M]). Since $S^{n-1}$ is simply connected, we see that each path connected component of $\partial \Omega$ must be diffeomorphic to $S^{n-1}$. Indeed, the restriction of $H$ to such a component serves as a diffeomorphism.

To proceed, we observe that from the assumption on $H$, it follows from implicit function theorem that for any $\xi \in S^{n-1}, H^{-1}(\xi)$ is a smooth one-dimensional submanifold of $\bar{\Omega}$. Moreover $H: \bar{\Omega} \rightarrow S^{n-1}$ is a smooth fiber bundle (see [M]). Fix a point $x_{0} \in \partial \Omega$, and let $\xi_{0}=H\left(x_{0}\right)$ and $\Gamma=H^{-1}\left(\xi_{0}\right)$. Then we have an exact sequence (see Theorem 6.7 of chapter VII in $[\mathrm{B}]$ )

$$
\pi_{n-1}\left(\Gamma, x_{0}\right) \rightarrow \pi_{n-1}\left(\bar{\Omega}, x_{0}\right) \rightarrow \pi_{n-1}\left(S^{n-1}, \xi_{0}\right) \rightarrow \pi_{n-2}\left(\Gamma, x_{0}\right) .
$$

If $n \geq 3$, then both $\pi_{n-1}\left(\Gamma, x_{0}\right)$ and $\pi_{n-2}\left(\Gamma, x_{0}\right)$ vanish. This shows that

$$
\pi_{n-1}\left(\bar{\Omega}, x_{0}\right) \cong \mathbb{Z}
$$

and hence $\bar{\Omega}$ is diffeomorphic to $\bar{B}_{2} \backslash B_{1}$. If $n=2$, then since $\pi_{1}\left(\Gamma, x_{0}\right)$ vanishes and $\pi_{0}\left(\Gamma, x_{0}\right)$ is finite, we see $\pi_{1}\left(\bar{\Omega}, x_{0}\right)$ is again isomorphic to $\mathbb{Z}$. This shows that $\bar{\Omega}$ must be diffeomorphic to $\bar{B}_{2} \backslash B_{1}$.

Now we are ready to prove the main theorem. 
Proof of Theorem 1. First if $\Omega=B_{1}$ or $B_{2} \backslash \bar{B}_{1}$, then $G(x)=x$ satisfies the assumption in Lemma 1 half of the theorem follows from Corollary 1 . On the other hand, assume for some $\Omega$ that we may find a smooth map $F$ satisfying (2). For $x \in \partial \Omega$, choose a base for the tangent space of $\partial \Omega$ at $x$, namely $e_{1}, \cdots, e_{n-1}$. Then

$$
\begin{aligned}
& \operatorname{rank}\left(F(x), \partial_{1} F(x), \cdots, \partial_{n} F(x)\right) \\
= & \operatorname{rank}\left(F(x), F_{*} e_{1}, \cdots, F_{*} e_{n-1}, F_{*} \nu\right) \\
= & \operatorname{rank}\left(F(x), F_{*} e_{1}, \cdots, F_{*} e_{n-1}\right)=n ;
\end{aligned}
$$

hence $F(x) \neq 0$ for any $x \in \partial \Omega$. Moreover, it follows from the fact

$$
\operatorname{rank}\left(F(x), \partial_{1} F(x), \cdots, \partial_{n} F(x)\right)=n \text { for any } x \in \bar{\Omega}
$$

that the zeroes of $F$ in $\Omega$ must be isolated. Hence $F$ has at most finitely many zeroes, say $x_{1}, \cdots, x_{m}$; here $m \geq 0$. For $\varepsilon>0$ small enough, let

$$
U=\Omega \backslash \bigcup_{i=1}^{m} \overline{B_{\varepsilon}\left(x_{i}\right)}
$$

if $m \geq 1$ and $U=\Omega$ if $m=0$. Then we have

$$
\operatorname{rank}\left(F(x), \partial_{1} F(x), \cdots, \partial_{n} F(x)\right)=n \text { for any } x \in \bar{U}
$$

and

$$
\operatorname{dim} \operatorname{span}\left\{F(x), \operatorname{im}\left(\left.F\right|_{\partial U}\right)_{*, x}\right\}=n \text { for any } x \in \partial U \text {. }
$$

Let

Then clearly

$$
H(x)=\frac{F(x)}{|F(x)|} \text { for } x \in \bar{U} .
$$

$$
\operatorname{rank}\left(\partial_{1} H(x), \cdots, \partial_{n} H(x)\right)=n-1 \text { for any } x \in \bar{U}
$$

and

$$
\operatorname{dimim}\left(\left.H\right|_{\partial U}\right)_{*, x}=n-1 \text { for any } x \in \partial U .
$$

It follows from Lemma 2 that $\bar{U}$ must be diffeomorphic to $\bar{B}_{2} \backslash B_{1}$, hence $m \leq 1$ and $\bar{\Omega}$ must be diffeomorphic to either $\bar{B}_{1}$ or $\bar{B}_{2} \backslash B_{1}$.

\section{ACKNOWLEDGMENT}

We would like to thank Professor Wei-Ming Ni for bringing the problem to our attention. We also thank Professor Peter Poláčik for his kind comments on the manuscript.

\section{REFERENCES}

[A] H. Amann. Existence and regularity for semilinear parabolic evolution equations. Scuola Morm. Sup. Pisa Cl. Sci. 11(4):593-696, 1984. MR0808425 (87h:34088)

[B] G. E. Bredon. Topology and geometry. Graduate Texts in Mathematics, 139. SpringerVerlag, New York, 1997. MR1700700 (2000b:55001)

[DP] E. N. Dancer and P. Poláčik. Realization of vector fields and dynamics of spatially homogeneous parabolic equations. Mem. AMS 140(668), 1999. MR.1618487 (99m:35125)

[H] D. Henry. Geometric theory of semilinear parabolic equations. Lecture notes in mathematics, Vol. 840, Springer-Verlag, New York, 1983. MR0610244 (83j:35084)

[M] J. W. Milnor. Topology from the differentiable viewpoint. Princeton Landmarks in Mathematics. Princeton University Press, Princeton, NJ, 1997. MR1487640 (98h:57051) 
[P1] P. Poláčik. Complicated dynamics in scalar semilinear parabolic equations in higher space dimension. Journal of Differential Equations 89(2):244-271, 1991. MR1091478 (92c:35063)

[P2] P. Poláčik. Imbedding of any vector field in a scalar semilinear parabolic equation. Proc. AMS. 115(4):1001-1008, 1992. MR.1089411 (92j:35099)

[P3] P. Poláčik. High-dimensional $\omega$-limit sets and chaos in scalar parabolic equations. J. Differential Equations 119(1):24-53, 1995. MR.1334487 (96h:35092)

[P4] P. Poláčik. Reaction-diffusion equations and realization of gradient vector fields. In International Conference on Differential Equations (Lisboa, 1995), 197-206. World Scientific Publishing, River Edge, NJ, 1998. MR1639355 (99j:35109)

[P5] P. Poláčik. Parabolic equations: asymptotic behavior and dynamics on invariant manifolds. Handbook on Dynamical Systems vol. 2: 835-883, B. Fiedler (ed.), Elsevier, Amsterdam, 2002. MR 1901067 (2003f:37150)

[PR] P. Poláčik and K. P. Rybakowski. Imbedding vector fields in scalar parabolic Dirichlet BVPs. Ann. Scuola Norm. Sup. Pisa Cl. Sci. (4) 22(4):737-749, 1995. MR1375317 (97a:35124)

[Pr] M. Prizzi. Perturbation of elliptic operators and complex dynamics of parabolic partial differential equations. Proc. Roy. Soc. Edinburgh Sect. A 130(2):397-418, 2000. MR 1750838 (2001e:35096)

[PrR1] M. Prizzi and K. P. Rybakowski. Some recent results on chaotic dynamics of parabolic equations. In Proceedings of the Conference "Topological Methods in Differential Equations and Dynamical Systems" (Kraków-Przegorzaly, 1996), no. 36, 231-235, 1998. MR 1661351

[PrR2] M. Prizzi and K. P. Rybakowski. Inverse problems and chaotic dynamics of parabolic equations on arbitrary spatial domains. J. Differential Equations 142(1):17-53, 1998. MR 1492876 (98k:35207)

[PrR3] M. Prizzi and K. P. Rybakowski. Complicated dynamics of parabolic equations with simple gradient dependence. Trans. Amer. Math. Soc. 350(8):3119-3130, 1998. MR1491875 (99a:35125)

[R] K. P. Rybakowski. The center manifold technique and complex dynamics of parabolic equations. In Topological methods in differential equations and inclusions (Montreal, PQ, 1994), Volume 472 of NATO Adv. Sci. Inst. Ser. C Math. Phys. Sci. 411-446. Kluwer Acad. Publ., Dordrecht, 1995. MR1368677 (97b:35095)

Department of Mathematics, Michigan State University, East Lansing, Michigan 48824

E-mail address: fhang@math.msu.edu

School of Mathematics, University of Minnesota, 127 Vincent Hall, 206 Church St. S.E., Minneapolis, Minnesota 55455

E-mail address: hqjiang@math.umn.edu 